\title{
Command Filter Backstepping Sliding Model Control for Lower-Limb Exoskeleton
}

\author{
Peng Yang, Gaowei Zhang, Jie Wang, Xiaozhou Wang, Lili Zhang, and Lingling Chen \\ School of Control Science and Engineering, Hebei University of Technology, Tianjin 300130, China \\ Correspondence should be addressed to Jie Wang; wangjie@hebut.edu.cn
}

Received 6 June 2017; Revised 26 August 2017; Accepted 17 September 2017; Published 13 November 2017

Academic Editor: Xuejun Xie

Copyright (C) 2017 Peng Yang et al. This is an open access article distributed under the Creative Commons Attribution License, which permits unrestricted use, distribution, and reproduction in any medium, provided the original work is properly cited.

A command filter adaptive fuzzy backstepping control strategy is proposed for lower-limb assisting exoskeleton. Firstly, the humanrobot model is established by taking the human body as a passive part, and a coupling torque is introduced to describe the interaction between the exoskeleton and human leg. Then, Vicon motion capture system is employed to obtain the reference trajectory. For the purpose of obviating the "explosion of complexity" in conventional backstepping, a second-order command filter is introduced into the sliding mode control strategy. The fuzzy logic systems (FLSs) are also applied to handle with the chattering problem by estimating the uncertainties and disturbances. Furthermore, the stability of the closed-loop system is proved based on the Lyapunov theory. Finally, simulation results are presented to illustrate the effectiveness of the control strategy.

\section{Introduction}

Recently, the exoskeleton is increasingly used for powerassisting in industrial [1,2], medical [3-5], and military [6,7] areas. The human operator provides locomotion intention and a small muscle strength while the robot can help human complete the desired action with a suitable torque through replicating the operator's movements [2]. The robot in such applications has been actively researched since 1990s, and some of them have been applied for various environments [8]. However, there are challenging problems in exoskeleton research such as the establishment of coupling model of human-robot system and designing of control strategy.

From the modeling point of view, it has been proved that some methods used now are effective such as NewtonEuler equations and Lagrange dynamics $[9,10]$. Based on aforementioned work, series of improvements have been proposed to increase the accuracy of human-robot system model. The inverse dynamic model is established and the singular points are avoided using damped least squares in [2]. In order to approximate the actual situation of human legs, a variety of musculoskeletal models are developed [11, 12]. The comparison between Hill-type and proportional model for human muscle is illustrated in [12], and Hill-type models are proved to be more appropriate.

Nowadays, there have been several published papers on control strategies of human-robot cooperative control [13-18]. For instance, a sensitivity amplification control is proposed in [19] which could track the desired trajectory by minimizing the interaction torque. In [20], a robust sliding mode controller is proposed to guarantee the stability in disturbance situation, and the boundary layer is introduced to reduce the chattering problem of sliding model control. Additionally, an adaptive sliding model control based on state observer is proposed in [21], which could update the controller parameters online to improve the safety of the system.

However, most of the control methods mentioned have limitations. In modeling, taking the human effects as disturbance [22], widely used in exoskeleton researches, is unreasonable. Human body is a part of the system obviously. Additional, the system has high-order features when taking the human body as a passive part [19]. Hence, the control strategies above are not available because of the "explosion of complexity" [23].

In this paper, a command filtered backstepping sliding model control equipped with FLSs is proposed based on 
a human-robot model. Compared with the analogous literature, the main contributions are summarized as follows:

(i) The proposed human-robot model is established by taking the human leg as a passive part and the coupling torque between human and robot is introduced. Compared with the model built in [19], this paper converts the transfer to the state space form and introduces uncertainties and disturbances which are more actual and complicated.

(ii) For the purpose of testing the controller performance, an experiment is implanted to obtain the actual trajectory of the hip-joint. Compared with the sine curve, the upper bound increases rapidly after a few derivative operations, which may cause the system uncontrollable.

(iii) The command filter backstepping sliding model control is proposed. By using the command filter, the analytical derivate is unnecessary and the "explosion of complexity" in the controller design process is avoided [24-26]. Besides, the problem mentioned in (ii) is solved. Furthermore, the FLSs are used to approximate the uncertainties and disturbances and provide real-time compensations for the system.

The paper is organized as follows: in Section 2, the high-order human-robot model is introduced based on the linearized models of human leg and exoskeleton. Then the reference trajectory of hip-joint is obtained through experiments. In Section 3, considering the demands of controller design, a model in state space form with uncertainties and disturbances is obtained. Based on that work, the humanrobot controller is designed with a command filter adaptive backstepping sliding mode and, by utilizing the Lyapunov theory, closed-loop system stability is analyzed. Simulation results are discussed in Section 4 and the conclusions are provided in Section 5.

\section{System Modeling and Trajectory Generation}

2.1. Dynamics of the Human-Robot System. As shown in Figure 1, the human-robot system can be expressed as a person wearing a lower-limb exoskeleton which provides a back support to assist hip-joint motion. By passing the leg's gravity to the waist, the muscle effort needed for human walking could be reduced to a low level.

In order to describe the system, an elementary model is used in this paper which consists of linearized onedegree-of-freedom (1-DOF) models for the human leg and the exoskeleton. In the process of modeling the physical interaction between the human and exoskeleton, a coupling torque, expressed as combination of a linear spring and a damper, is introduced.

Then, the ideal dynamics of the human-robot system are given as follows [19]:

$$
\begin{gathered}
I_{h} \ddot{\theta}_{h}(t)+b_{h} \dot{\theta}_{h}(t)+k_{h} \theta_{h}(t)=\tau_{h}(t)-\tau_{c}(t) \\
I_{e} \ddot{\theta}_{e}(t)+b_{e} \dot{\theta}_{e}(t)+k_{e} \theta_{e}(t)=\tau_{e}(t)+\tau_{c}(t),
\end{gathered}
$$

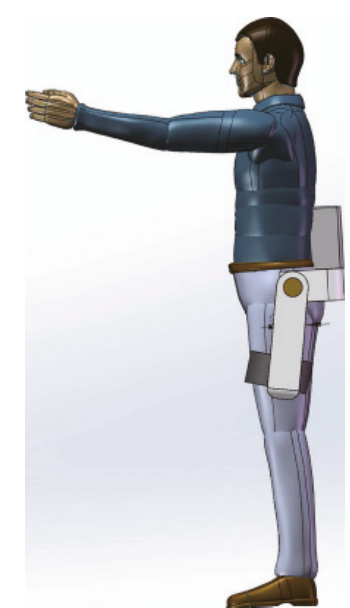

FIgURE 1: 1-DOF lower-limb exoskeleton.
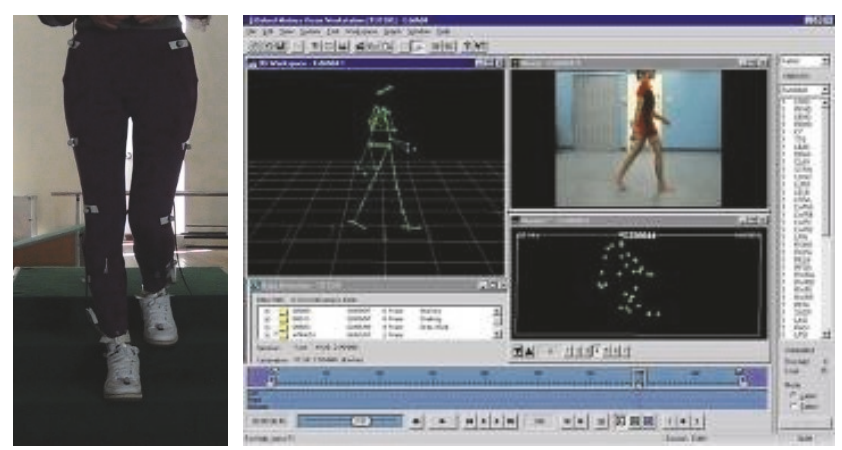

FIGURE 2: Vicon motion capture system.

where $I_{h}, b_{h}, k_{h}$ are, respectively, the moment of inertia, joint damping coefficient, and joint stiffness coefficient of the human leg; $\theta_{h}(t)$ is the hip-joint angle; $\tau_{h}(t)$ is the net muscle torque acting on the joint. $I_{e}, b_{e}, k_{e}$ are, respectively, the moment of inertia, joint damping coefficient, and joint stiffness coefficient of the exoskeleton; $\theta_{e}(t)$ is the exoskeleton joint angle; $\tau_{e}(t)$ is the actuator torque.

And the coupling torque is defined as follows:

$$
b_{c}\left(\dot{\theta}_{h}(t)-\dot{\theta}_{e}(t)\right)+k_{c}\left(\theta_{h}(t)-\theta_{e}(t)\right)=\tau_{c}(t),
$$

where $b_{c}, k_{c}$ are, respectively, the equivalent damping coefficient and stiffness coefficient of the interaction torque; $\tau_{c}$ is the coupling torque.

2.2. Trajectory Generation. The main way that the exoskeleton helps human complete the locomotion is tracking the human gait cycle. So a reasonable desired position trajectory is an essential factor for testing the model and controller. An experimenter (girl aged 25 years with mass of $52 \mathrm{~kg}$ and stature of $165 \mathrm{~cm}$ ) volunteered to participate in the gait experiment with Vicon motion capture system, device provided by National Research Center for Rehabilitation Technical Aids (Figure 2).

Special trackers are fixed at the particular marks on the experimenter which can be captured by cameras distributed 
TABLE 1: Parameter identification of the hip joint trajectory.

\begin{tabular}{lc}
\hline$\theta_{0}$ & $9.50(9.46,9.55)$ \\
$a_{1}$ & $-18.74(-18.8,-18.7)$ \\
$a_{2}$ & $1.957(1.9,2.0)$ \\
$a_{3}$ & $0.7747(0.72,0.83)$ \\
$w_{n}$ & $3.775(3.772,3.778)$ \\
$b_{1}$ & $-8.97(-9.0,-8.9)$ \\
$b_{2}$ & $-5.026(-5.09,-4.96)$ \\
$b_{3}$ & $0.9843(0.93,1.04)$ \\
\hline
\end{tabular}

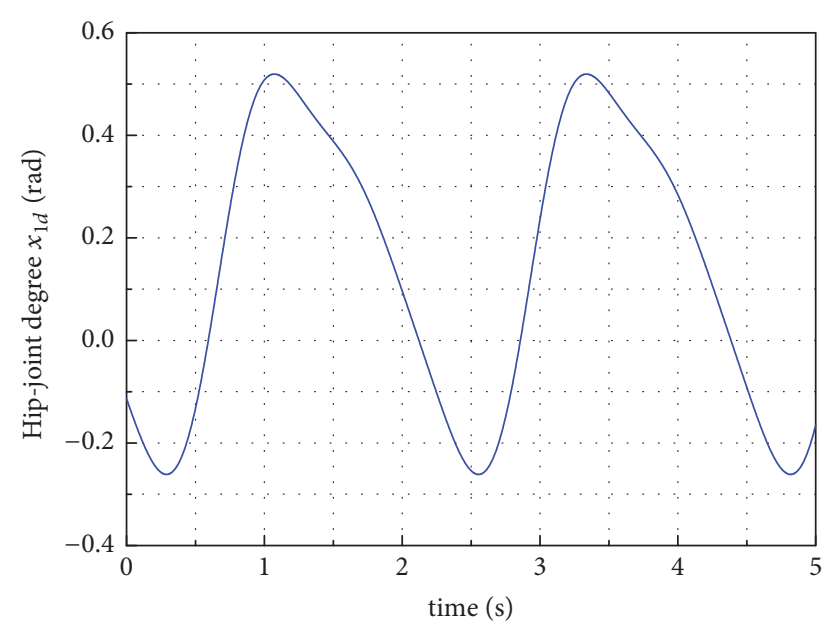

FIgURE 3: The actual hip-joint trajectory obtained by experiment.

in reasonable location in experiment space. The information from different cameras are combined, and the actual hipjoint angle signals are obtained through data processing. From the considerable data obtained, the most reliable, representative, and authentic data is selected and reorganized. To ensure the smoothness of the trajectory, the Fourier series is introduced to describe the actual gait cycle. Note that the human locomotion satisfies the smooth characteristics.

Mathematical expression of the desired trajectory can be expressed as follows [14]:

$$
\theta_{r}=\theta_{0}+\sum_{k=1}^{N}\left(a_{k} \sin \left(k w_{n} t\right)+b_{k} \cos \left(k w_{n} t\right)\right)
$$

where $\theta_{0}$ is the initial value of $\theta_{2} ; a_{k}$ and $b_{k}$ are the sine and cosine amplitudes of Fourier series; $w_{n}$ is the fundamental frequency; $k$ is the harmonic order.

The parameters of the trajectory are obtained by the curve fit toolbox of MATLAB. In order to approximate the actual curve and simplify the calculation process, fundamental frequency to third harmonics, that is, $k=3$ in (3), of the Fourier series is used in this paper. The parameters are illustrated in Table 1.

According to (3) and the parameters given in Table 1, a reliable hip-joint trajectory (expressed as $x_{1 d}$ ) can be obtained and shown in Figure 3.
The control objectives for the human-robot system are illustrated as follows:

(i) An adaptive controller for high-order human-robot system is designed, such that the position of human leg $x_{1}$ and exoskeleton $x_{3}$ can track the actual trajectory $x_{1 d}$ obtained from experiment.

(ii) The prescribed output tracking error $\tilde{x}_{1}=x_{1}-x_{1 c}$ is always bounded. Besides when uncertainties and disturbances exist in the system, the tracking error can converge to a neighborhood of the origin in a short time.

\section{Controller Design}

3.1. System Description and Control Strategy. For the exoskeleton, the human leg is a passive part and fulfills the locomotion with the interaction torque between the human and robot. For the dynamics shown in (1), when replacing $\tau_{c}$ with (2), the system is given by

$$
\begin{gathered}
I_{h} \ddot{\theta}_{h}+\left(b_{h}+b_{c}\right) \dot{\theta}_{h}+\left(k_{h}+k_{c}\right) \theta_{h}-b_{c} \dot{\theta}_{e}-k_{c} \theta_{e}=\tau_{h} \\
I_{e} \ddot{\theta}_{e}+\left(b_{e}+b_{c}\right) \dot{\theta}_{e}+\left(k_{e}+k_{c}\right) \theta_{e}-b_{c} \dot{\theta}_{h}-k_{c} \theta_{h}=\tau_{e} .
\end{gathered}
$$

Note that all the states and the torques are time-varying variables and time flags are omitted for convenience.

Taking the lump uncertainties, parametric/unmodeled uncertainties as well as the external disturbances, into account, (4) can be transformed into state space as

$$
\begin{aligned}
\dot{x}_{1}= & x_{2} \\
\dot{x}_{2} & \\
= & I_{h}^{-1}\left[\tau_{h}-\left(k_{c}+k_{h}\right) x_{1}-\left(b_{c}+b_{h}\right) x_{2}+k_{c} x_{3}+b_{c} x_{4}\right] \\
& +\Delta_{1} \\
\dot{x}_{3}= & x_{4} \\
\dot{x}_{4} & \\
= & I_{e}^{-1}\left[\tau_{e}+k_{c} x_{1}+b_{c} x_{2}-\left(k_{c}+k_{e}\right) x_{3}-\left(b_{c}+b_{e}\right) x_{4}\right] \\
& +\Delta_{2},
\end{aligned}
$$

where $x_{1}=\theta_{h}, x_{2}=\dot{\theta}_{h}, x_{3}=\theta_{e}, x_{4}=\dot{\theta}_{e}$ are the state vector of the system; the lump uncertainties in human-robot system are

$$
\begin{aligned}
\Delta_{1} & =I_{h}^{-1}\left(-\Delta\left(k_{c}+k_{h}\right) x_{1}-\Delta\left(b_{c}+b_{h}\right) x_{2}+\Delta k_{c} x_{3}\right. \\
& \left.+\Delta b_{c} x_{4}\right)+d_{1} \\
\Delta_{2} & =I_{e}^{-1}\left(\Delta k_{c} x_{1}+\Delta b_{c} x_{2}-\Delta\left(k_{c}+k_{e}\right) x_{3}\right. \\
& \left.-\Delta\left(b_{c}+b_{e}\right) x_{4}\right)+d_{2} .
\end{aligned}
$$

Note that the specific parameters of human leg are hard to be measured and the actuator of the exoskeleton includes mechanical errors which cannot be described precisely. Hence, the uncertainties of the system existed and are inevitable. 
Considering that the system has high-order features, a backstepping sliding model method is introduced to solve the complex problem with a recursive form [27]. To avoid the "explosion of complexity" in the controller design process, a second-order nonlinear command filter is employed in this paper. The command filter ensures that the desired command and its derivative satisfy the same magnitude and rate constrains [24].

In order to handle the system chattering problem caused by the uncertainties and disturbances, fuzzy logic systems (FLSs) are equipped to estimate the upper bounds of the lump uncertainties. FLSs provide real-time compensations for the human-robot system to reduce the switching items of the sliding model control.

Being equipped with second-order command filter and FLSs, a backstepping sliding mode control strategy for the human-robot system with uncertainties and disturbances is proposed in this paper.

3.2. Basic Assumptions and FLS. Some reasonable and useful assumptions are given at first which ensure the stability of the system.

Assumption 1. There exist constants $c_{1}, c_{2}>0$, such that the inequality $\left|\Delta_{1}\right|<c_{1},\left|\Delta_{2}\right|<c_{2}$ holds.

Assumption 2 (Lipschitz). For $\forall x_{1}, x_{2} \in R^{n}, \exists L \in R^{+}$, the inequality holds as follows:

$$
\left|F\left(x_{1}\right)-F\left(x_{2}\right)\right| \leq L\left\|x_{1}-x_{2}\right\|
$$

where $\left\|x_{1}-x_{2}\right\|$ represents the 2 -norm of $\left(x_{1}-x_{2}\right)$. And all the desired commands $x_{i d}, \dot{x}_{i d}(i=1,2,3,4)$ satisfy the Lipschitz continuity.

The design of the FLS consists of two steps. First, the fuzzy rule base should be made up as follows:

$$
\begin{aligned}
& R^{l}: \text { if } x_{1} \text { is } A_{1}^{l} \text { and } x_{2} \text { is } A_{2}^{l} \text { and... and } x_{n} \text { is } A_{n}^{l} \\
& \text { then } \widehat{f}(x) \text { is } B^{l},
\end{aligned}
$$

where $A_{i}^{l}$ and $B_{l}$ are fuzzy sets in $R, l=1,2, \ldots, N ; i=$ $1,2, \ldots, n$.

The second step is defuzzification. Center average defuzzification operator is applied in this paper which can be expressed as

$$
\widehat{f}(x)=\frac{\sum_{l=1}^{N} \bar{f}_{l} \prod_{i=1}^{n} u_{A_{i}^{l}}\left(x_{i}\right)}{\sum_{l=1}^{N}\left[\prod_{i=1}^{n} u_{A_{i}^{l}}\left(x_{i}\right)\right]}
$$

where $\bar{f}_{l}=\max _{f \in R} u_{B^{l}}(f)$, and $u_{A_{i}^{l}}\left(x_{i}\right), u_{B^{l}}$ are the membership functions.

Define the fuzzy basis vector as

$$
\varphi(x)=\frac{\prod_{i=1}^{n} u_{A_{i}^{l}}\left(x_{i}\right)}{\sum_{l=1}^{N}\left[\prod_{i=1}^{n} u_{A_{i}^{l}}\left(x_{i}\right)\right]} .
$$

Denote $\theta^{T}=\left[\bar{f}_{1}, \bar{f}_{2}, \ldots, \bar{f}_{N}\right]$ and $\varphi(x)=\left[\varphi_{1}(x)\right.$, $\left.\varphi_{2}(x), \ldots, \varphi_{n}(x)\right]^{T}$; then the FLS can be expressed as

$$
\widehat{f}(x)=\theta^{T} \varphi(x) .
$$

The optimal parameter $\theta^{* T}$ can be defined by

$$
\theta^{* T}=\arg \min _{\theta \in R^{n}}\left\{\sup _{x \in R^{n}}\left|f(x)-\theta^{* T} \varphi(x)\right|\right\} .
$$

Lemma 3 (Wang [28]). For $\forall f(x)$, which is continuous function and defined over a compact $\Omega$, for any a constant $\varepsilon \in R^{+}$, there exist an FLS and a parameter $\theta^{*}$ such that

$$
\sup _{x \in \Omega}\left|f(x)-\theta^{* T} \varphi(x)\right| \leq \varepsilon .
$$

3.3. Controller Design. Consider the characteristics of the human-robot system, a backstepping sliding model control with second-order command filter is proposed in this paper. The controller design process is shown in this section.

The output tracking errors and compensated tracking errors of the subsystems are defined, respectively, as

$$
\begin{aligned}
& \tilde{x}_{i}=x_{i}-x_{i c}, \quad i=1,2,3,4 . \\
& z_{i}=\tilde{x}_{i}-\xi_{i}, \quad i=1,2,3,4,
\end{aligned}
$$

where $x_{i}$ and $x_{i c}$ represent the system states and filtered commands of the $i$ th subsystem, respectively.

The $\xi_{i}(i=1,2,3,4)$ signals can be obtained by

$$
\begin{aligned}
& \dot{\xi}_{1}=-k_{1} \xi_{1}+\left(x_{2 c}-x_{2 d}\right)+\xi_{2} \\
& \dot{\xi}_{2}=-k_{2} \xi_{2}+I_{h}^{-1} k_{c}\left(x_{3 c}-x_{3 d}\right)+I_{h}^{-1} k_{c} \xi_{3} \\
& \dot{\xi}_{3}=-k_{3} \xi_{3}+\left(x_{4 c}-x_{4 d}\right)+\xi_{4} \\
& \dot{\xi}_{4}=-k_{4} \xi_{4}+I_{e}^{-1}\left(\tau_{e c}-\tau_{e d}\right)
\end{aligned}
$$

with $\xi_{i}(0)=0$.

Remark 4. Equation (14) is used to achieve the filtering value $\xi_{i}$ which are designed to compensate the errors caused by the command filters. Note that they can be computed with integrating processes to avoid the differential operations.

For the purpose of eliminating the "explosion of complexity," a second-order nonlinear command filter is designed to calculate $x_{i c}, \dot{x}_{i c}$. So the command filter is shown as follows:

$$
\begin{aligned}
& {\left[\begin{array}{c}
\dot{r}_{i, 1} \\
\dot{r}_{i, 2}
\end{array}\right]=\left[\begin{array}{c}
r_{i, 2} \\
-2 \zeta \omega_{n} r_{i, 2}-\omega_{n}^{2}\left(r_{i, 1}-x_{i d}\right)
\end{array}\right]} \\
& {\left[\begin{array}{c}
x_{i c} \\
\dot{x}_{i c}
\end{array}\right]=\left[\begin{array}{c}
r_{i, 1} \\
r_{i, 2}
\end{array}\right],}
\end{aligned}
$$

where $\omega_{n}$ is the natural frequency of the filter and typically satisfies $\omega_{n}>k_{i}, i=(1,2,3,4)$ to ensure the tracking accuracy. $\zeta \in(0,1]$ is the damping ratio of the filter system. 
$x_{i d},(i=2,3,4)$ are the virtual control signals and $x_{1 d}$ is the desired trajectory.

The filter initial conditions are $x_{i c}(0)=0, \dot{x}_{i c}(0)=0$. Every command filter is designed to compute the filtered commands without differential operation. Furthermore, $x_{i c}$ will track $x_{i d}$ by choosing the suitable parameters.

In order to find the optimal parameters of the FLSs, the adaptive laws are chosen for $\widehat{\theta}_{1}$ and $\widehat{\theta}_{2}$ as follows:

$$
\begin{aligned}
& \dot{\hat{\theta}}_{1}=\gamma_{1} z_{2} \varphi_{1} \\
& \dot{\hat{\theta}}_{2}=\gamma_{2} z_{4} \varphi_{2},
\end{aligned}
$$

where $\gamma_{1}, \gamma_{2}$ are the positive adaptive coefficients.

Considering the compensating errors and the closed-loop dynamics, the virtual law can be defined as follows:

$$
\begin{aligned}
x_{2 d} & =-k_{1} \tilde{x}_{1}+\dot{x}_{1 c} \\
x_{3 d} & =k_{c}^{-1}\left[I_{h}\left(-k_{2} \tilde{x}_{2}-z_{1}+\dot{x}_{2 c}-\widehat{\theta}_{1}^{T} \varphi_{1}-\eta_{1} \text { sats }\left(z_{2}\right)\right)\right. \\
& \left.-\tau_{h}+\left(k_{c}+k_{h}\right) x_{1}+\left(b_{c}+b_{h}\right) x_{2}-b_{c} x_{4}\right] \\
x_{4 d} & =-k_{3} \tilde{x}_{3}-k_{c} I_{h}^{-1} z_{2}+\dot{x}_{3 c} \\
\tau_{e d} & =I_{e}\left[-k_{4} \tilde{x}_{4}+\dot{x}_{4}-z_{3}-\widehat{\theta}_{2}^{T} \varphi_{2}-\eta_{2} \operatorname{sats}\left(z_{4}\right)\right] \\
& -k_{c} x_{1}-b_{c} x_{2}+\left(k_{c}+k_{e}\right) x_{3}+\left(b_{c}+b_{e}\right) x_{4},
\end{aligned}
$$

where $\widehat{\theta}_{1}, \widehat{\theta}_{2}$ are the estimated values of optimal parameters and $\varphi_{1}, \varphi_{2}$ are the basis vectors of FLSs. $k_{i}>0,(i=1,2,3,4)$ are control gains specified by the designer. $\eta_{1}, \eta_{2}$ are constants that ensure that the inequality $\eta_{1}>\left|\varepsilon_{1}\right|, \eta_{2}>\left|\varepsilon_{2}\right|$ holds.

The sats $(x)$ is the switching function that satisfies

$$
\text { sats }(x)= \begin{cases}1, & x>x_{\Delta} \\ k x, & |x| \leq x_{\Delta} \\ -1, & x<-x_{\Delta} .\end{cases}
$$

\subsection{Stability Analysis}

Theorem 5. For the system illustrated in (5), there exist a range of values for the gains $k_{1}, k_{2}, k_{3}, k_{4}$, and the adaptive coefficients $\gamma_{1}, \gamma_{2}$, such that the tracking error $\tilde{x}_{1}$ and compensation errors $z_{i}(i=1,2,3,4)$ can converge to zero with the compensations provided by FLSs.

Proof. The tracking error $\tilde{x}_{i}$ and the compensated tracking error $z_{i}$ are given first.

$$
\begin{aligned}
\dot{\tilde{x}}_{1} & =\dot{x}_{1}-\dot{x}_{1 c}=x_{2}-x_{2 c}+x_{2 c}-x_{2 d}+x_{2 d}-\dot{x}_{1 c} \\
& =\tilde{x}_{2}+\left(x_{2 c}-x_{2 d}\right)+x_{2 d}-\dot{x}_{1 c}=-k_{1} \tilde{x}_{1}+\tilde{x}_{2} \\
& +\left(x_{2 c}-x_{2 d}\right)
\end{aligned}
$$

$$
\begin{aligned}
\dot{\tilde{x}}_{2} & =\dot{x}_{2}-\dot{x}_{2 c}=I_{h}^{-1}\left[\tau_{h}-\left(k_{c}+k_{h}\right) x_{1}-\left(b_{c}+b_{h}\right) x_{2}\right. \\
& \left.+k_{c}\left(x_{3}-x_{3 c}+x_{3 c}-x_{3 d}+x_{3 d}\right) b_{c} x_{4}\right]+\Delta_{1}-\dot{x}_{2 c} \\
& =I_{h}^{-1}\left[\tau_{h}-\left(k_{c}+k_{h}\right) x_{1}-\left(b_{c}+b_{h}\right) x_{2}+k_{c} x_{3 d}\right. \\
& \left.+k_{c} \tilde{x}_{3}+b_{c} x_{4}\right]+\Delta_{1}+I_{h}^{-1} k_{c}\left(x_{3 c}-x_{3 d}\right)-\dot{x}_{2 c} \\
& =-k_{2} \tilde{x}_{2}-z_{1}+I_{h}^{-1} k_{c} \tilde{x}_{3}+I_{h}^{-1} k_{c}\left(x_{3 c}-x_{3 d}\right) \\
& +\widetilde{\theta}_{1}^{T} \varphi_{1}+\varepsilon_{1}-\eta_{1} \operatorname{sats}\left(z_{2}\right) \\
\dot{\tilde{x}}_{3} & =\dot{x}_{3}-\dot{x}_{3 c}=x_{4}-x_{4 c}+x_{4 c}-x_{4 d}+x_{4 d}-\dot{x}_{3 c} \\
& =\tilde{x}_{4}+\left(x_{4 c}-x_{4 d}\right)+x_{4 d}-\dot{x}_{3 c}=-k_{3} \tilde{x}_{3}+\tilde{x}_{4} \\
& +\left(x_{4 c}-x_{4 d}\right)-k_{c} I_{h}^{-1} z_{2} \\
\dot{\tilde{x}}_{4} & =\dot{x}_{4}-\dot{x}_{3 c}=I_{e}^{-1}\left[\tau_{e}-\tau_{e c}+\tau_{e c}-\tau_{e d}+\tau_{e d}+k_{c} x_{1}\right. \\
& \left.+b_{c} x_{2}-\left(k_{c}+k_{e}\right) x_{3}-\left(b_{c}+b_{e}\right) x_{4}\right]+\Delta_{2}-\dot{x}_{3 c} \\
& =I_{e}^{-1}\left[\widetilde{\tau}_{e}-\left(\tau_{e c}-\tau_{e d}\right)+\tau_{e d}+k_{c} x_{1}+b_{c} x_{2}\right. \\
& \left.-\left(k_{c}+k_{e}\right) x_{3}-\left(b_{c}+b_{e}\right) x_{4}\right]+\Delta_{2}-\dot{x}_{3 c}=-k_{4} \tilde{x}_{4} \\
& -z_{3}+I_{e}^{-1}\left[\widetilde{\tau}_{e}-\left(\tau_{e c}-\tau_{e d}\right)\right]+\widetilde{\theta}_{2}^{T} \varphi_{2}+\varepsilon_{2} \\
& -\eta_{2} \operatorname{sats}\left(z_{4}\right) .
\end{aligned}
$$

Due to the fact that $z_{i}=\tilde{x}_{i}-\xi_{i}$ for $i=1,2,3,4$, the dynamics of compensated tracking error can be expressed as follows:

$$
\begin{aligned}
\dot{z}_{1}= & \dot{\tilde{x}}_{1}-\dot{\xi}_{1} \\
= & -k_{1} \tilde{x}_{1}+\tilde{x}_{2}+\left(x_{2 c}-x_{2 d}\right) \\
& -\left[-k_{1} \xi_{1}+\left(x_{2 c}-x_{2 d}\right)+\xi_{2}\right]=-k_{1} z_{1}+z_{2} \\
\dot{z}_{2}= & \dot{\tilde{x}}_{2}-\dot{\xi}_{2} \\
= & -k_{2} \tilde{x}_{2}-z_{1}+I_{h}^{-1} k_{c} \tilde{x}_{3}+I_{h}^{-1} k_{c}\left(x_{3 c}-x_{3 d}\right)+\widetilde{\theta}_{1}^{T} \varphi \\
& +\varepsilon_{1}-\eta_{1} \operatorname{sats}\left(z_{2}\right) \\
& -\left[-k_{2} \xi_{2}+I_{h}^{-1} k_{c}\left(x_{3 c}-x_{3 d}\right)+I_{h}^{-1} k_{c} \xi_{3}\right] \\
= & -k_{2} z_{2}-z_{1}+I_{h}^{-1} k_{c} z_{3}+\widetilde{\theta}_{1}^{T} \varphi+\varepsilon_{1}-\eta_{1} \text { sats }\left(z_{2}\right) \\
\dot{z}_{3}= & \dot{\tilde{x}}_{3}-\dot{\xi}_{3} \\
= & -k_{3} \tilde{x}_{3}+\tilde{x}_{4}+\left(x_{4 c}-x_{4 d}\right)-k_{c} I_{h}^{-1} z_{2} \\
& -\left[-k_{3} \xi_{3}+\left(x_{4 c}-x_{4 d}\right)+\xi_{4}\right] \\
= & -k_{3} z_{3}+z_{4}-k_{c} I_{h}^{-1} z_{2} \\
\dot{z}_{4}= & \dot{\tilde{x}}_{4}-\dot{\xi}_{4} \\
= & -k_{4} \tilde{x}_{4}-z_{3}+I_{e}^{-1}\left(\tilde{\tau}_{e}-\left(\tau_{e c}-\tau_{e d}\right)\right)+\widetilde{\theta}_{2}^{T} \varphi_{2}+\varepsilon_{2} \\
& -\eta_{2} \operatorname{sats}\left(z_{4}\right)-\left[-k_{4} \xi_{4}+I_{e}^{-1}\left(\tau_{e c}-\tau_{e d}\right)\right] \\
= & -k_{4} z_{4}-z_{3}+\widetilde{\theta}_{2}^{T} \varphi_{2}+\varepsilon_{2}-\eta_{2} \operatorname{sats}\left(z_{4}\right)+I_{e}^{-1} \widetilde{\tau}_{e}, \\
& \sigma_{2}
\end{aligned}
$$

where $\widetilde{\theta}_{1}=\theta_{1}^{*}-\widehat{\theta}_{1}$ and $\widetilde{\theta}_{2}=\theta_{2}^{*}-\widehat{\theta}_{2}$. 
Remark 6. Note the fact that the command $\tau_{e c}$ will directly output to control plant, so the conclusion is easily obtained that $\tau_{e}=\tau_{e c}$.

Then, define the control Lyapunov function for the closed-loop system as

$$
V(t)=\frac{1}{2}\left(\sum_{i=1}^{4} z_{i}^{2}+\gamma_{1}^{-1} \widetilde{\theta}_{1}^{T} \widetilde{\theta}_{1}+\gamma_{2}^{-1} \widetilde{\theta}_{2}^{T} \widetilde{\theta}_{2}\right) .
$$

Then the time derivative of the Lyapunov function (24) becomes

$$
\dot{V}(t)=\sum_{i=1}^{4} z_{i} \dot{z}_{i}-\gamma_{1}^{-1} \widetilde{\theta}_{1}^{T} \dot{\hat{\theta}}_{1}-\gamma_{2}^{-1} \widetilde{\theta}_{2}^{T} \dot{\hat{\theta}}_{2} .
$$

Substitute $\dot{z}_{i}$ with (23):

$$
\begin{aligned}
& \dot{V}(t)=z_{1}\left(-k_{1} z_{1}+z_{2}\right)+z_{2}\left(-k_{2} z_{2}-z_{1}+I_{h}^{-1} k_{c} z_{3}\right. \\
& \left.+\widetilde{\theta}_{1}^{T} \varphi+\varepsilon_{1}-\eta_{1} \text { sats }\left(z_{2}\right)\right)+z_{3}\left(-k_{3} z_{3}+z_{4}\right. \\
& \left.\quad-k_{c} I_{h}^{-1} z_{2}\right)+z_{4}\left(-k_{4} z_{4}-z_{3}+\widetilde{\theta}_{2}^{T} \varphi_{2}+\varepsilon_{2}\right. \\
& \left.\quad-\eta_{2} \text { sats }\left(z_{4}\right)\right)-\gamma_{1}^{-1} \widetilde{\theta}_{1}^{T} \dot{\hat{\theta}}_{1}-\gamma_{2}^{-1} \widetilde{\theta}_{2}^{T} \dot{\hat{\theta}}_{2} .
\end{aligned}
$$

The simplification can be written as

$$
\begin{aligned}
\dot{V}(t)= & -k_{1} z_{1}^{2}-k_{2} z_{2}^{2}-k_{3} z_{3}^{2}-k_{4} z_{4}^{2} \\
& +z_{2}\left(\widetilde{\theta}_{1}^{T} \varphi+\varepsilon_{1}-\eta_{1} \text { sats }\left(z_{2}\right)\right) \\
& +z_{4}\left(\widetilde{\theta}_{2}^{T} \varphi_{2}+\varepsilon_{2}-\eta_{2} \operatorname{sats}\left(z_{4}\right)\right)-\gamma_{1}^{-1} \widetilde{\theta}_{1}^{T} \dot{\hat{\theta}}_{1} \\
& -\gamma_{2}^{-1} \widetilde{\theta}_{2}^{T} \dot{\hat{\theta}}_{2} .
\end{aligned}
$$

Replace $\dot{\hat{\theta}}_{1}, \dot{\hat{\theta}}_{2}$ with the adaptive law (16)

$$
\begin{aligned}
\dot{V}(t)= & -k_{1} z_{1}^{2}-k_{2} z_{2}^{2}-k_{3} z_{3}^{2}-k_{4} z_{4}^{2} \\
& +z_{2}\left(\varepsilon_{1}-\eta_{1} \operatorname{sats}\left(z_{2}\right)\right)+z_{4}\left(\varepsilon_{2}-\eta_{2} \text { sats }\left(z_{4}\right)\right) \\
\leq & -k_{1} z_{1}^{2}-k_{2} z_{2}^{2}-k_{3} z_{3}^{2}-k_{4} z_{4}^{2}+\left|z_{2}\right|\left(\varepsilon_{1}-\eta_{1}\right) \\
& +\left|z_{4}\right|\left(\varepsilon_{2}-\eta_{2}\right) .
\end{aligned}
$$

Let $\eta_{1}>\left|\varepsilon_{1}\right|, \eta_{2}>\left|\varepsilon_{2}\right|$; define the tracking error vector as $z=\left[z_{1}, z_{2}, z_{3}, z_{4}\right]$; then

$$
\dot{V}(t) \leq-k_{1} z_{1}^{2}-k_{2} z_{2}^{2}-k_{3} z_{3}^{2}-k_{4} z_{4}^{2} \leq-k_{\min }\|z\|^{2},
$$

where $k_{\min }=\min \left\{k_{1}, k_{2}, k_{3}, k_{4}\right\}$. According to (29), the error vector $z$ is uniformly bounded, and $\lim _{t \rightarrow \infty} V(t)=V(\infty)$. When integrating both sides of inequality (29), then

$$
V(\infty)-V(0) \leq-k_{\min } \int_{0}^{\infty}\|z\|^{2} d t
$$

That is

$$
k_{\min } \int_{0}^{\infty}\|z\|^{2} d t \leq V(0)-V(\infty) .
$$

TABLE 2: Parameters of the human-robot system.

\begin{tabular}{lc}
\hline PARM & Value \\
\hline$I_{e}$ & $0.01178 \mathrm{~kg} \mathrm{~m}^{2}$ \\
$b_{e}$ & $0.345 \mathrm{~N} \mathrm{~m} \mathrm{~s} / \mathrm{rad}$ \\
$k_{e}$ & $0.339 \mathrm{~N} \mathrm{~m} / \mathrm{rad}$ \\
$I_{h}$ & $3.38 \mathrm{~kg} \mathrm{~m}$ \\
$b_{h}$ & $3.5 \mathrm{~N} \mathrm{~m} \mathrm{~s} / \mathrm{rad}$ \\
$k_{h}$ & $54.7 \mathrm{~N} \mathrm{~m} / \mathrm{rad}$ \\
$b_{c}$ & $9.47 \mathrm{~N} \mathrm{~m} \mathrm{~s} / \mathrm{rad}$ \\
$k_{c}$ & $1905 \mathrm{~N} \mathrm{~m} / \mathrm{rad}$ \\
\hline
\end{tabular}

TABLE 3: The parameters of the controller.

\begin{tabular}{lc}
\hline PARM & Value \\
\hline$k_{1}$ & 50 \\
$k_{2}$ & 50 \\
$k_{3}$ & 50 \\
$k_{4}$ & 50 \\
$\eta_{1}$ & 0.05 \\
$\eta_{2}$ & 0.05 \\
$\gamma_{1}$ & 150 \\
$\gamma_{2}$ & 30 \\
$w_{n}$ & 500 \\
$\varsigma$ & 0.9 \\
\hline
\end{tabular}

According to Barbalat Lemma proposed in [29], when the time variable $t$ tends to infinity, the error vector $z$ tends to zero.

The block diagram of controller design is shown in Figure 4 .

\section{Simulation Results}

In this section, a simulation of an 1-DOF lower-limb exoskeleton is established. All the parameters of the 1-DOF exoskeleton are illustrated in Table 2.

The PARM is short for parameter, and the notation is omitted for convenience through the paper as long as special notation is not required.

Remark 7. All the parameters are cited in [19]. The parameters, just for calculating, are obtained from real experiment and useful for controller simulation.

The lump uncertainties are chosen as follows:

$$
\begin{aligned}
\frac{\Delta k_{c}}{k_{c}} & =\frac{\Delta b_{c}}{b_{c}}=\frac{\Delta k_{h}}{k_{h}}=\frac{\Delta b_{h}}{b_{h}}=0.1, \\
d_{1} & =d_{2}=0.2 \sin (t) .
\end{aligned}
$$

To ensure the stability of the system, the specific parameters of the controller are tuned in a trial-and-error procedure and shown in Table 3.

The simulation results are given in Figures 5-10. It can be seen that the controller designed can guarantee the uncertainties and external disturbances. 

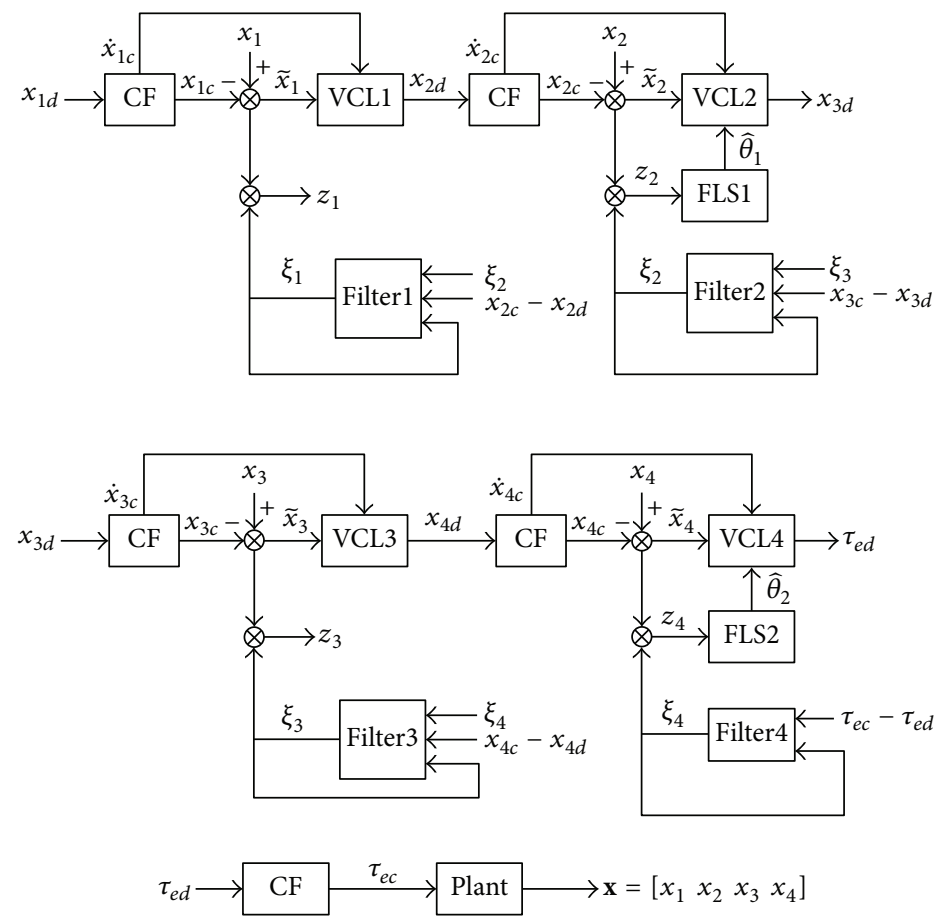

Figure 4: Block diagram of the controller design. (CF represents the command filter defined by (15); VCL represents the virtual control law of the four subsystems defined by (17)-(20); FLS represents the fuzzy logic system.)

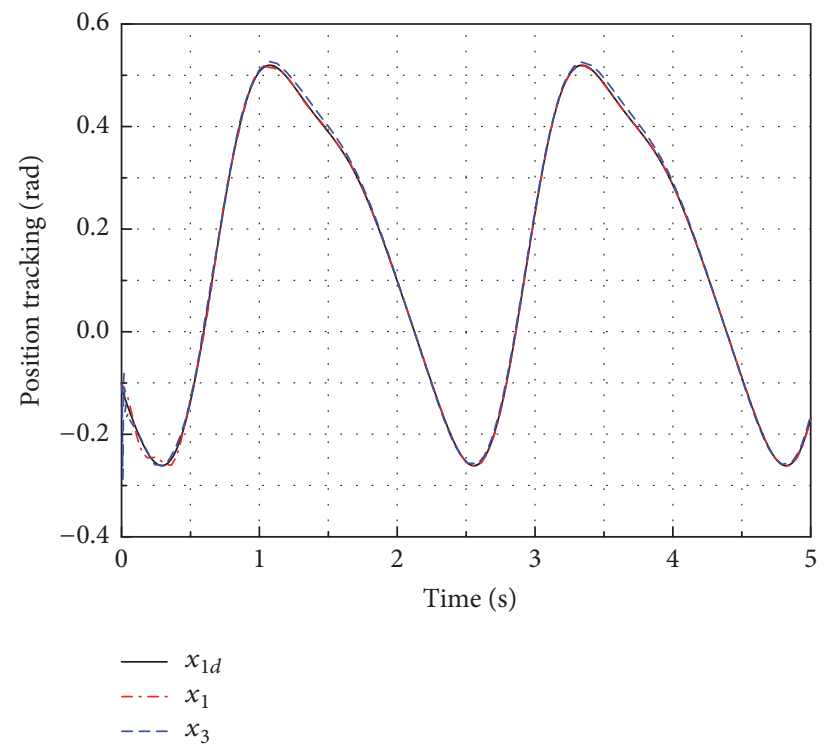

FIGURE 5: Position tracking of human-robot system.

Figures 5 and 6 illustrate the position tracking and the tracking errors of the human-robot system. In Figure 5, the desired trajectory $x_{1 d}$ is represented as solid line, the human leg position $x_{1}$ is shown as dash-dotted line, and the position of exoskeleton $x_{3}$ is described as dashed line. Just as it is shown, the controlled plant can steadily track the hip curve in a satisfactory way. Figure 6 shows the errors of the trajectory tracking. It is easy to get that the proposed command filtered fuzzy adaptive backstepping controller could make the errors

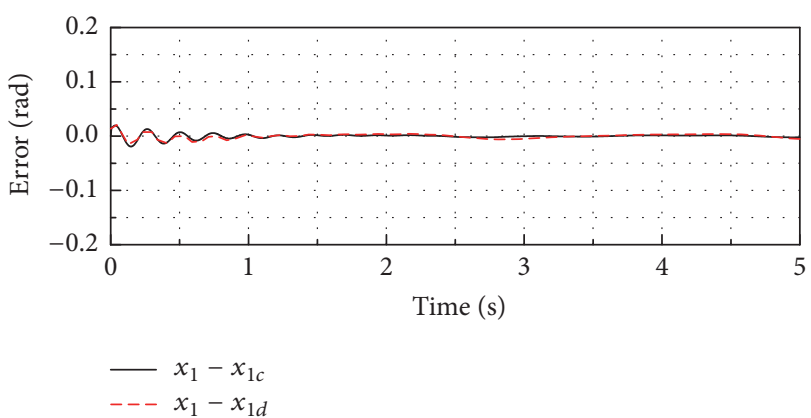

FIGURE 6: Tracking errors of human-robot system.

finally kept in the neighborhood of the origin from the figure. However there are still differences between the two errors because of the filtered errors.

Figures 7 and 8 show the virtual control signals $x_{i d}$ as solid line and the filtered commands $x_{i c}$, which are produced by passing the virtual control signals through the secondorder filter, as the dashed line. As expected, the control signals of all the subsystems are smooth and bounded. Moreover, the virtual control signals and the corresponding filtered commands satisfy the same magnitude, rate, and bandwidth constrains, which is different from the traditional first-order linear filter. The closed-loop tracking errors could converge to a tiny range around the origin in a very short time (about 0.1 s) just as shown in Figure 9.

Figure 10 illustrates the system's lump uncertainties and estimated values obtained by FLSs. The lump uncertainties $\Delta_{1}, \Delta_{2}$ are expressed as solid lines and the estimates $\Delta_{e 1}, \Delta_{e 2}$ 

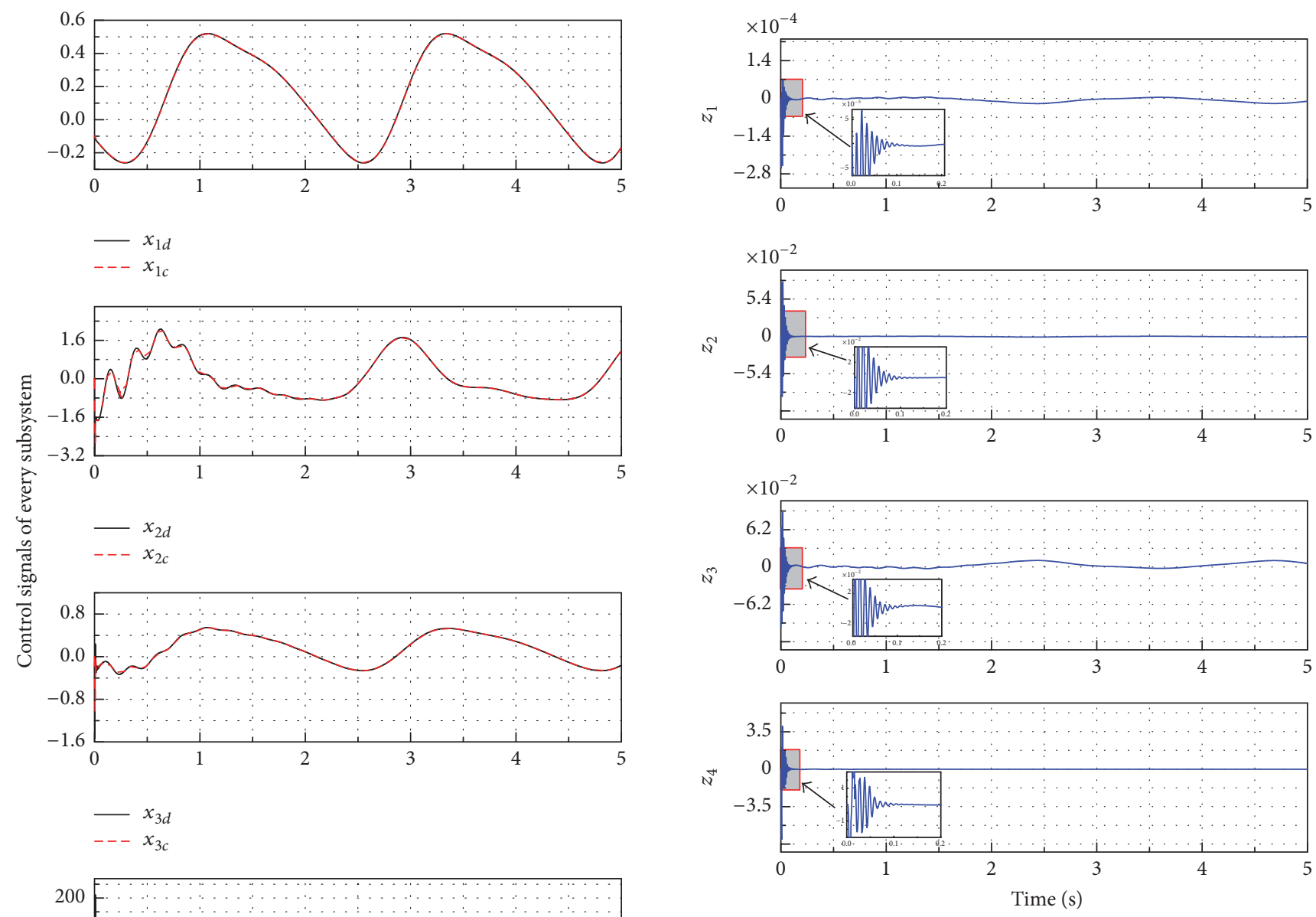

FIgURE 9: Compensated tracking error.

are represented as dashed lines. It can be seen that the estimated value (dash) reaching the real value (solid) in less than $0.2 \mathrm{~s}$. Therefrom, upper bounds of the switch terms in sliding model control could be much smaller because of the compensations provided by FLSs.

All the signals remain bounded in a reasonable range during the process. Obviously, the proposed control strategy with command filters and FLSs can be a suitable method for lower-limb exoskeleton.

\section{Conclusion}

A human-robot cooperative control strategy based on a convincing high-order model is proposed for a lower-limb assisting exoskeleton. A second-order command filter backstepping method is employed to determine the time derivatives of virtual control signals without differential operations. The FLSs are used to approximate the uncertainties and disturbances and compensate the system timely. In addition, the stability of the system is proved based on the Lyapunov theory. Finally, simulation results are presented to verify the effectiveness of the proposed command filter adaptive fuzzy control strategy.

Future work will focus on the performance of the control strategy in actual experiment and the filtering errors should 


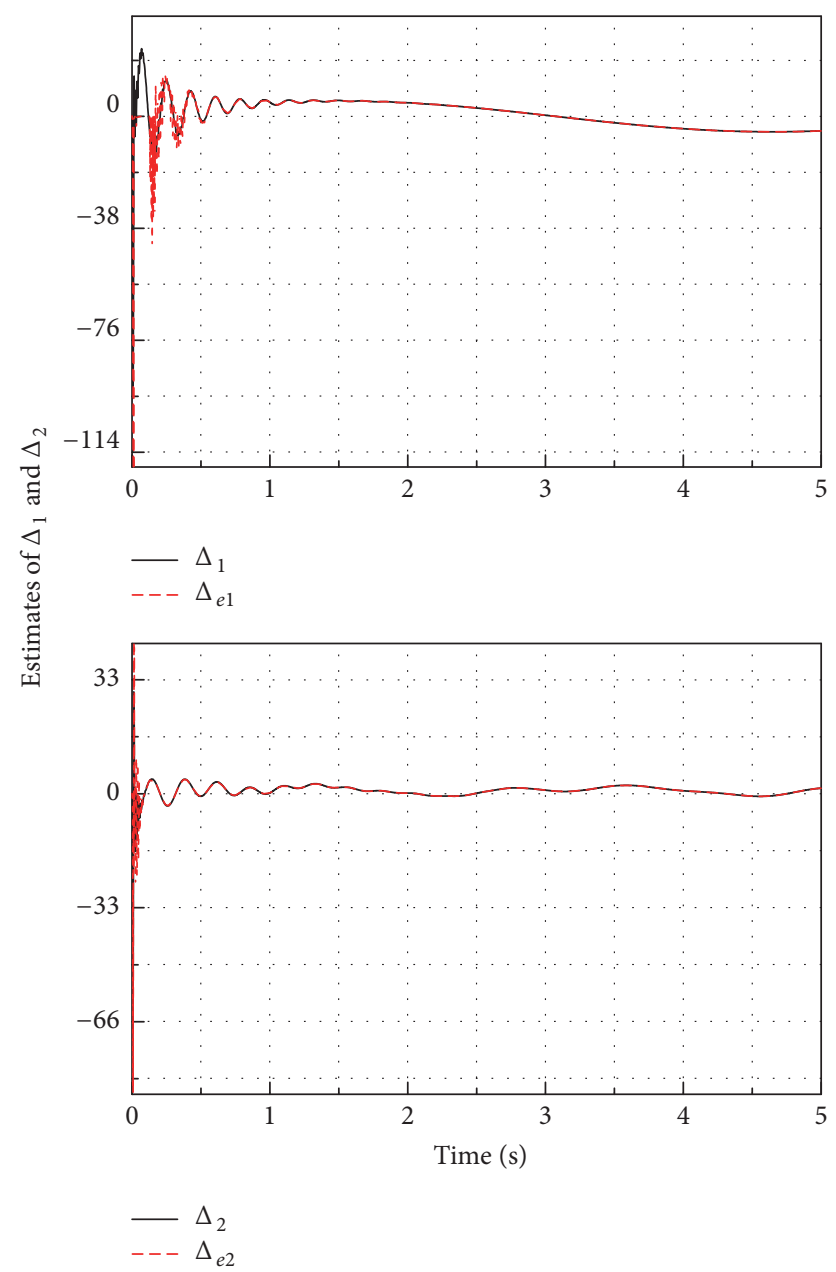

FIGURE 10: The estimate of the uncertainties and disturbances with the FLSs.

be proved to converge rigorously. Besides, further research on the state constrain control for high-order nonlinear systems [30] is also needed.

\section{Conflicts of Interest}

The authors declare that they have no conflicts of interest.

\section{Acknowledgments}

This work is supported by National Natural Science Foundation (NNSF) of China under Grants 61703134, 61503118, and 61703135, the Natural Science Foundation of Hebei Province (nos. F2015202150; F2017202119; F2016202327), the Natural Science Foundation of Tianjin (no. 17JCQNJC04400), and Foundation of Hebei Educational Committee (nos. QN2015068; ZD2016071).

\section{References}

[1] K. S. Stadler, R. Altenburger, E. Schmidhauser et al., "Robomate an exoskeleton for industrial use-concept and mechanical design," in Advances in Cooperative Robotics, pp. 806-813, 2017.
[2] H. Lee, B. Lee, W. Kim, M. Gil, J. Han, and C. Han, "Humanrobot cooperative control based on pHRI (Physical HumanRobot Interaction) of exoskeleton robot for a human upper extremity," International Journal of Precision Engineering and Manufacturing, vol. 13, no. 6, pp. 985-992, 2012.

[3] R. Lu, Z. Li, C.-Y. Su, and A. Xue, "Development and learning control of a human limb with a rehabilitation exoskeleton," IEEE Transactions on Industrial Electronics, vol. 61, no. 7, pp. 37763785, 2014.

[4] A. J. del-Ama, Á. Gil-Agudo, J. L. Pons, and J. C. Moreno, "Hybrid FES-robot cooperative control of ambulatory gait rehabilitation exoskeleton," Journal of NeuroEngineering and Rehabilitation, vol. 11, no. 1, article 27, 2014.

[5] S. Hussain, S. Q. Xie, and P. K. Jamwal, "Robust nonlinear control of an intrinsically compliant robotic gait training orthosis," IEEE Transactions on Systems, Man, and Cybernetics: Systems, vol. 43, no. 3, pp. 655-665, 2013.

[6] R. Bogue, "Robotic exoskeletons: A review of recent progress," Industrial Robot: An International Journal, vol. 42, no. 1, pp. 510, 2015.

[7] R. Steger, K. Sung Hoon, and H. Kazerooni, "Control scheme and networked control architecture for the Berkeley Lower Extremity Exoskeleton (BLEEX)," in Proceedings of the IEEE International Conference on Robotics and Automation (ICRA '06), pp. 3469-3476, May 2006.

[8] W. Meng, Q. Liu, Z. Zhou, Q. Ai, B. Sheng, and S. S. Xie, "Recent development of mechanisms and control strategies for robotassisted lower limb rehabilitation," Mechatronics, vol. 31, pp. 132-145, 2015.

[9] M. O. Ajayi, K. Djouani, and Y. Hamam, "Rhythmic trajectory design and control for rehabilitative walking in patients with lower limb disorder," International Journal of Humanoid Robotics, vol. 13, no. 4, Article ID 1650006, 2016.

[10] E. Piña-Martínez and E. Rodriguez-Leal, "Inverse modeling of human knee joint based on geometry and vision systems for exoskeleton applications," Mathematical Problems in Engineering, vol. 2015, Article ID 145734, 14 pages, 2015.

[11] D. J. Farris, J. L. Hicks, S. L. Delp, and G. S. Sawicki, "Musculoskeletal modelling deconstructs the paradoxical effects of elastic ankle exoskeletons on plantar-flexor mechanics and energetics during hopping," Journal of Experimental Biology, vol. 217, no. 22, pp. 4018-4028, 2014.

[12] D. Ao, R. Song, and J. Gao, "Movement performance of human-robot cooperation control based on EMG-driven hilltype and proportional models for an ankle power-assist exoskeleton robot," IEEE Transactions on Neural Systems and Rehabilitation Engineering, vol. 25, no. 8, pp. 1125-1134, 2017.

[13] Y. Long, Z. J. Du, and W. D. Wang, "RBF neural network with genetic algorithm optimization based sensitivity amplification control for exoskeleton," Journal of Harbin Institute of Technology, vol. 47, no. 7, pp. 26-30, 2015.

[14] S. Mefoued, "A second order sliding mode control and a neural network to drive a knee joint actuated orthosis," Neurocomputing, vol. 155, pp. 71-79, 2015.

[15] Y. Long, Z.-J. Du, W.-D. Wang, and W. Dong, "Robust sliding mode control based on GA optimization and CMAC compensation for lower limb exoskeleton," Applied Bionics and Biomechanics, vol. 2016, Article ID 5017381, 13 pages, 2016.

[16] Q. Guo, S. Li, and D. Jiang, "A lower extremity exoskeleton: human-machine coupled modeling, robust control design, simulation, and overload-carrying experiment," Mathematical 
Problems in Engineering, vol. 2015, Article ID 905761, 15 pages, 2015.

[17] Y. Zhu, T. Zheng, H. Jin, J. Yang, and J. Zhao, "Double closedloop cascade control for lower limb exoskeleton with elastic actuation," Technology and Health Care, vol. 24, no. 1, pp. S113S122, 2016.

[18] S. Balasubramanian and J. He, "Adaptive control of a wearable exoskeleton for upper-extremity neurorehabilitation," Applied Bionics and Biomechanics, vol. 9, no. 1, pp. 99-115, 2012.

[19] U. Nagarajan, G. Aguirre-Ollinger, and A. Goswami, "Integral admittance shaping: A unified framework for active exoskeleton control," Robotics and Autonomous Systems, vol. 75, pp. 310-324, 2016.

[20] M. H. Rahman, T. Kittel-Ouimet, M. Saad, J.-P. Kenné, and P. S. Archambault, "Development and control of a robotic exoskeleton for shoulder, elbow and forearm movement assistance," Applied Bionics and Biomechanics, vol. 9, no. 3, pp. 275-292, 2012.

[21] H.-B. Kang, "Adaptive control of 5 DOF upper-limb exoskeleton robot with improved safety," ISA Transactions, vol. 52, no. 6, pp. 844-852, 2013.

[22] S. Mohammed, W. Huo, J. Huang, H. Rifaï, and Y. Amirat, "Nonlinear disturbance observer based sliding mode control of a human-driven knee joint orthosis," Robotics and Autonomous Systems, vol. 75, pp. 41-49, 2016.

[23] Y. ji, H. Zhou, and Q. Zong, "ISPS-modular command-filtered adaptive back-stepping control of non-linearly parameterized pure-feedback systems," Transactions of the Institute of Measurement and Control, vol. 38, no. 2, pp. 232-239, 2016.

[24] Y. Wang, L. Cao, S. Zhang, X. Hu, and F. Yu, “Command filtered adaptive fuzzy backstepping control method of uncertain nonlinear systems," IET Control Theory \& Applications, vol. 10, no. 10, pp. 1134-1141, 2016.

[25] W. Yan, J. Huang, and D. Xu, "Adaptive command-filtered backstepping control for linear induction motor via projection algorithm," Mathematical Problems in Engineering, vol. 2016, Article ID 4720126, 13 pages, 2016.

[26] B. Niu, Y. Liu, G. Zong, Z. Han, and J. Fu, "Command filterbased adaptive neural tracking controller design for uncertain switched nonlinear output-constrained systems," IEEE Transactions on Cybernetics, vol. 47, no. 10, pp. 3160-3171, 2017.

[27] B. Tian, Z. Zuo, and H. Wang, "Leader-follower fixed-time consensus of multi-agent systems with high-order integrator dynamics," International Journal of Control, vol. 90, no. 7, pp. 1420-1427, 2017.

[28] L. X. Wang, Adaptive Fuzzy Systems and Control, Prentice-Hall, Englewood Cliffs, NJ, USA, 1994.

[29] K. Lu, Y. Xia, Z. Zhu, and M. V. Basin, "Sliding mode attitude tracking of rigid spacecraft with disturbances," Journal of The Franklin Institute, vol. 349, no. 2, pp. 413-440, 2012.

[30] L. Liu and X. Yang, "Robust adaptive state constraint control for uncertain switched high-order nonlinear systems," IEEE Transactions on Industrial Electronics, vol. 64, no. 10, pp. 81088117, 2017. 


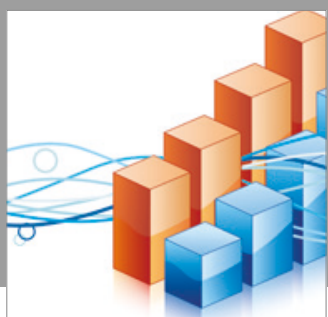

Advances in

Operations Research

vatersals

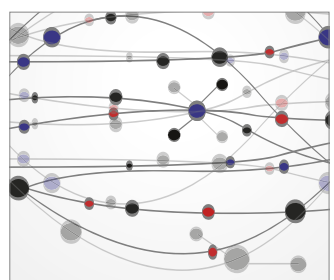

\section{The Scientific} World Journal
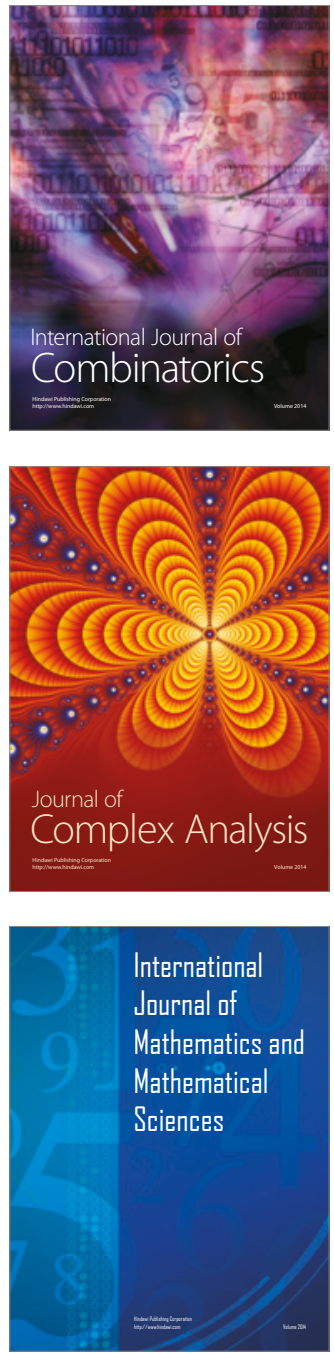
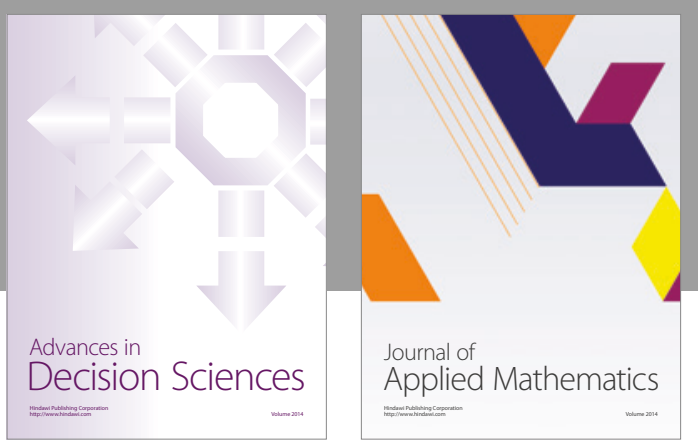

Algebra

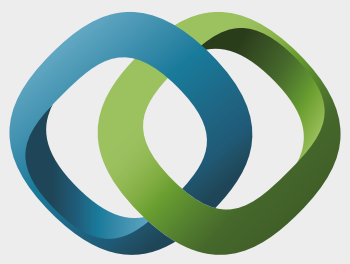

\section{Hindawi}

Submit your manuscripts at

https://www.hindawi.com
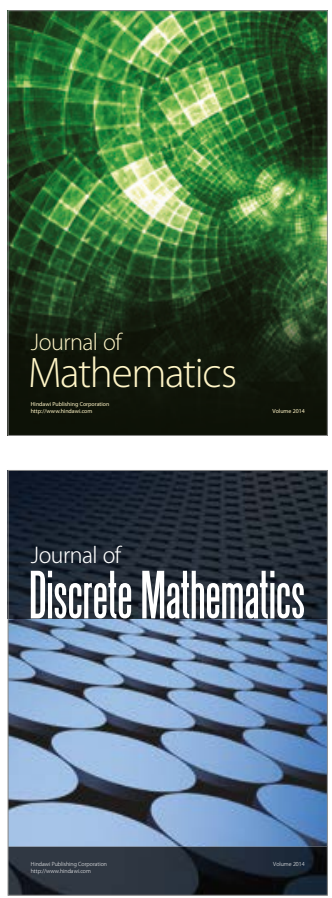

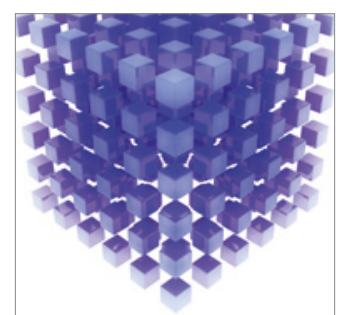

Mathematical Problems in Engineering
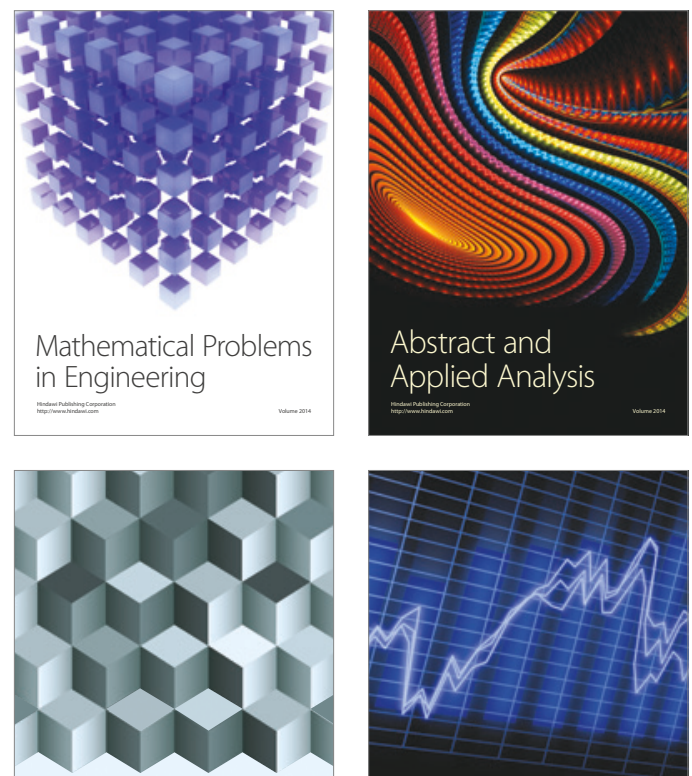

Journal of

Function Spaces

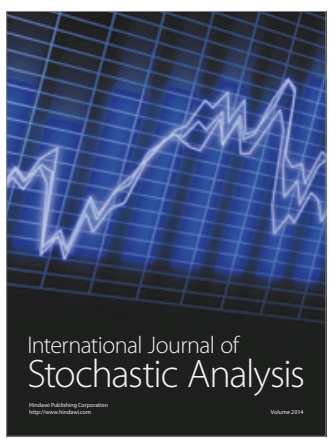

Probability and Statistics
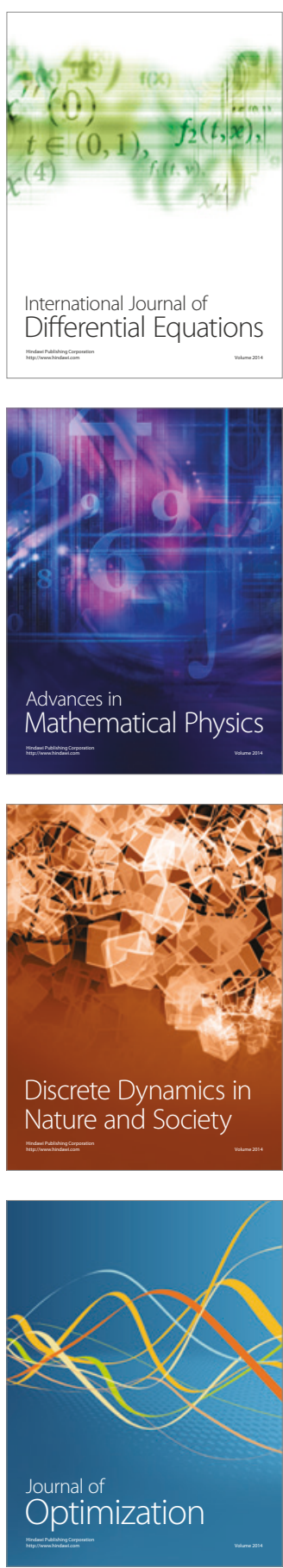